\title{
Engineering Laboratory/Factory-Specific Phage- Resistant Strains Of Escherichia Coli By Mutagenesis And Selection
}

\section{Ya-Ping Xue ( $\nabla$ xyp@zjut.edu.cn )}

Zhejiang University of Technology https://orcid.org/0000-0003-0370-2502

\section{Qi Shen}

Zhejiang University of Technology Chaohui Campus: Zhejiang University of Technology

\section{Xiao-Ting Zhou}

Zhejiang University of Technology

\section{Qian Guo}

Zhejiang University of Technology Chaohui Campus: Zhejiang University of Technology

\section{Yu-Guo Zheng}

Zhejiang University of Technology Chaohui Campus: Zhejiang University of Technology

\section{Research Article}

Keywords: phage-resistant, E. coli, mutagenesis, laboratory/factory-specific, contamination

Posted Date: April 28th, 2021

DOI: https://doi.org/10.21203/rs.3.rs-415349/v1

License: (9) This work is licensed under a Creative Commons Attribution 4.0 International License. Read Full License 


\section{Abstract}

Objective: The phage contamination is always a serious problem during operation of Escherichia coli ( $E$. coli). The present study aims to engineer laboratory/factory phage-resistant strains of $E$. coli based on mutagenesis and selection.

Results: The laboratory or factory derived phage was added into a tube containing mutagenized $E$. coli, resulted in achieving respective phage-resistant $E$. coli strain. Interestingly, the resistance spectrum of generated phage-resistant strains was not limited to the phage they encountered, which may make these E. coli mutants more resistant to phage contamination. When operation of a resistant strain at the position where the corresponding phage derived from, the phage contamination was completely prevented. There was no significant difference in heterogeneous protein production between the parental strain and the phage-resistant strains. Importantly, the whole process of mutagenesis and selection only required one day.

Conclusions: This practical method for engineering laboratory/factory-specific phage-resistant strains may have great potential in resuming $E$. coli operation in laboratory and factory when the phage contamination outbreaks.

\section{Introduction}

Escherichia coli $(E . c o l i)$ is a gram-negative bacterium that is the most frequently used host for producing recombinant proteins in laboratory and industry (Baeshen et al. 2015). Comparing with other expression hosts, the $E$. coli has several advantages such as a fast-growing rate, high heterogeneous protein yields, and well-annotated genomes (Wang et al. 2017). However, a serious problem of application of $E$. coli is that it is vulnerable to phage attack (Baltz 2018). The contamination of phage not only makes current batch useless, but also may influence the following batches in industrial manufacture. In the case of contamination in laboratory, the phage may spread throughout the laboratory.

There are some tips for preventing phage contaminations. Among them, a good hygiene condition for operation of $E$. coli is the most important, but phage infections still happen time to time. Maintaining the E. coli at low temperatures can suppress the phage growth (Los 2012). However, this strategy is always accompanied by a low yield of the desired bio-product. While several phage-resistant $E$. coli strains were engineered (Li et al. 2019), none of them was widely used. Therefore, the development of a practical method of preventing the phage infections may have great potential in both laboratories and factories.

Considering that species of phage causing contaminations are dynamically changing over time and location, laboratory/factory-specific phage-resistant strains of $E$. coli may be helpful in decreasing the chances of phage infections. However, rational engineering of an $E$. coli strain with the resistance to a newly encountered phage requires adequate time and effort (Labrie et al. 2010; Rostol and Marraffini 2019). An alternative is through mutagenesis and selection. Likun et al have reported that an antiphage $E$. coli mutant producing of L-threonine can be obtained by ARTP mutagenesis (Cheng et al. 2020). It 
remains unknown whether this strategy is strain or phage-specific, and the performance of antiphage $E$. coli mutant in a real environment has not been evaluated. Herein, we systematically investigated this method by using four species of phages and three $E$. coli strains, and the strain-phage interactions were also revealed.

\section{Materials And Methods}

\section{Strains, plasmids, enzymes, and reagents}

E. coli BL21(DE3) was used as an expression host (Thermo Fisher Scientific, Carlsbad, USA). pET-28a (+) vector was purchased from Novagen (Darmstadt, Germany). Vector pPGAP-GFPuv and strain expressing a nitrilase from Acidovorax facilis (Mut3) were constructed and preserved in our laboratory (Shen et al. 2020; Zhang et al. 2014). ClonExpress II One Step Cloning Kit was obtained from Vazyme Biotech (Nanjing, China). Low melting point (LMP) agarose gel was purchased from Shanghai Shenggong Biological Engineering Co., Ltd. (Shanghai, China). The codon-optimized Haemophilus ducreyi nicotinamide phosphoribosyltransferase with a C-terminal six histidine tag was synthesized by Beijing Qingke biocompany (Beijing, China). The nicotinamide phosphoribosyltransferase catalyzes the conversion of phosphoribosyl diphosphate into nicotinamide mononucleotide (Wang et al. 2006).

\section{Plaque assay}

Four supernatants of suspicious phage-contaminated $E$. coli broth from laboratory or factory were used as phage stocks. Serial 10-fold dilutions were performed in Luria-Bertani (LB) medium and $10 \mathrm{ul}$ of the diluted stock samples were incubated with 100 ul of log-phase $E$. coli BL21(DE3) for 20 min. This mixture was transferred into a tube containing $3.5 \mathrm{ml}$ of cooled down LB LMB agarose (0.35\%), and all contents of the tube were poured onto a LB plate. Plates were incubated at $37^{\circ} \mathrm{C}$ overnight. The plate has appropriate numbers of plaque was used for counting. The phage amount in a phage stock was calculated as: phage forming units (PFU)/ul= number of plaques counted/ (dilution factor of the plate for counting $\times 10)$.

\section{Construction of E. coli strain expressing the Haemophilus ducreyi nicotinamide phosphoribosyltransferase}

The linearized pET-28a vector was amplified from pET-28a by reverse PCR using primers FP-pET-28a and RP-pET-28a (all primers used for construction of plasmids were listed in Table 1). The sequence of Haemophilus ducreyi nicotinamide phosphoribosyltransferas was amplified from the synthesized product using primers FP1-NAMPT and RP1-NAMPT. The amplified Haemophilus ducreyi nicotinamide phosphoribosyltransferas was inserted into the linearized pET-28a vector to give pET-28a-NAMPT by recombination using ClonExpress II One Step Cloning Kit according to the manufacturer. The representative strain transformed by pET-28a-NAMPT was assigned as Hd NAMPT.

Table 1. Primers used for construction of vectors 


\begin{tabular}{|ll|}
\hline Primer & Primer sequence (5'-3') \\
\hline FP1-NAMPT & tttaagaaggagatataccaATGGATAACCTGCTGAATTATAGTAGTCG \\
\hline RP1-NAMPT & aggccgctgctgtgatgatgTTAATGGTGATGATGATGATGCAG \\
\hline FP-pET-28a & CATCATCACAGCAGCGGCC \\
\hline RP-pET-28a & TGGTATATCTCCTTCTTAAAGTTAAACAAA \\
FP1-GFP & tttaagaaggagatataccaATGGCTAGCAAAGGAGAAGAACTT \\
RP1-GFP & aggccgctgctgtgatgatgTTATTTGTAGAGCTCATCCATGCC \\
\hline
\end{tabular}

\section{Construction of $E$. coli strain expressing a green fluorescent protein variant}

The sequence of a green fluorescent protein variant optimized for excitation by UV light (GFPuv) was amplified from pPGAP-GFPuv using primers FP1-GFP and RP1-GFP. The amplified GFPuv gene was inserted into the linearized pET-28a vector to give pET-28a-GFPuv by recombination using ClonExpress II One Step Cloning Kit according to the manufacturer. The representative strain transformed by pET-28aGFPuv was assigned as GFP.

\section{Expression of recombinant proteins}

E. coli strains were seed in LB medium with kanamycin at $37^{\circ} \mathrm{C}$. Upon the optical density at $600 \mathrm{~nm}$ $\left(\mathrm{OD}_{600}\right)$ reached $0.6-0.8$, cells were induced with $0.1 \mathrm{mM}$ of IPTG at $28^{\circ} \mathrm{C}$ for $10 \mathrm{~h}$. After washing with potassium phosphate buffer (PBS), cells were disrupted for $20 \mathrm{~min}$ by intermittent sonication on ice and subsequently centrifugated at 12,000 rpm for removing cell debris. 5 ul of supernatant was subjected to a 4-12\% GenScript SurePAGE gel (Nanjing, China). After electrophoresis, the gel was strained by Coomassie Blue R-250.

\section{Determination of the relative fluorescence unit}

After IPTG induction, cells from $0.5 \mathrm{ml}$ culture broth were washed with PBS three times and were then transferred into 96-well plates for measurement of fluorescence values using an excitation wavelength of $395 \mathrm{~nm}$ and an emission wavelength of $506 \mathrm{~nm}$.

\section{Mutagenesis and selection}

The mutant library was generated by the atmospheric and room temperature plasma (ARTP) mutation system (ARTP-M, Wuxi, China). The radio-frequency power input, the stand-off distance, and the plama jet temperature were $120 \mathrm{w}, 2.0 \mathrm{~mm}$, and $<40^{\circ} \mathrm{C}$, respectively. $20 \mathrm{ul}$ of log-phase $E$. coli cells were treated for $40 \mathrm{~s}$. All the treated cells were transferred into $1 \mathrm{ml} \mathrm{LB}$ medium and incubated at $37^{\circ} \mathrm{C}$ with shaking for $5.5 \mathrm{~h} .5 \mathrm{ul}$ of phage stock was then added into the medium containing the mutagenized $E$. coli.

\section{Determination of sensitivity of $E$. coli strains to phages}


Strains were cultured into tubes containing $3 \mathrm{ml}$ of LB media with kanamycin at $37^{\circ} \mathrm{C}$ until the $\mathrm{OD}_{600}$ reached $0.8-0.9,5 \mathrm{ul}$ of phage stock was then added into the broth, $\mathrm{OD}_{600}$ was measured in indicated time points.

\section{Statistical analysis}

The one-way ANOVA test was applied for statistical analysis using the GraphPad Prism software v7.00. Results are presented as mean $\pm S D(n=3)$. Statistical significance was defined as $*: p<0.05$, and $* *$ : $\mathrm{p}<0.01$.

\section{Results}

\section{Generating laboratory/factory-specific phage-resistant strains of E. coli by mutagenesis and selection}

The plaque assay was performed for confirming the PFU in the supernatants of suspicious phagecontaminated $E$. coli broth (while phage1, phage2, and phage3 were derived from different laboratories, phage4 was collected from a factory). The PFU in these supernatants ranged from $1.4 \times 10^{6}$ to $2.7 \times 10^{7}$ (Table 2). It is notable that the plaque size formed by phage 1 and phage 2 was apparently smaller than phage3 and phage4 (Fig. 1A).

Table 2

The PFU in phage stocks

\begin{tabular}{|ll|}
\hline Phage stock & PFU \\
\hline phage 1 & $2.5 \times 10^{6}$ \\
\hline phage2 & $2.7 \times 10^{7}$ \\
\hline phage3 & $5.2 \times 10^{6}$ \\
phage4 & $1.4 \times 10^{6}$ \\
\hline
\end{tabular}

To generate resistant strains, supernatants containing each species of phage were added into ARTP mutagenized $H d$ NAMPT. To make the method more practical, $5 \mathrm{ul}$ of supernatants were added for screening resistant strains regardless of the PFU in them. The workflow for generation laboratory/factoryspecific phage-resistant strains was shown in Fig. 1B. The $\mathrm{OD}_{600}$ of cultures became clear in $4 \mathrm{~h}$ upon the addition of the phage-contaminated cell supernatants for both parental and mutagenized cells (Fig. 1C). However, the $\mathrm{OD}_{600}$ values rebounded in $16 \mathrm{~h}$ of incubation for the ARTP mutagenized $E$. coli cells (Fig. 1C). The strains achieved through the treatment with phage1, phage2, phage3, and phage4 were named $H d 1, H d 2, H d 3$, and $H d 4$, respectively. Adding the corresponding species of phage into $H d 1$, $H d 2$, $H d 3$, and $H d 4$ during the period of logarithmic growth did not cause cell lysis (Fig. 1D), indicating these strains acquired immunity to the phages that they have encountered. Interestingly, the resistance spectrum of generated phage-resistant strains was not limited to the phage they encountered (Fig. 1E-H). 
For example, $H d 1$ has the ability to reach normal cell densities $\left(\mathrm{OD}_{600}\right.$ larger than 1.0$)$ after $16 \mathrm{~h}$ of adding phage2, phage3, or phage4 (Fig. 1E). However, the cell growth pattern was different for $\mathrm{Hd} 1$ after treated with different phages, as phage 2 and phage 4 but not phage 3 suppressed the $H d 1$ proliferation at $4 \mathrm{~h}$. Only for $H d 2$, adding neither phage stocks decreased the $\mathrm{OD}_{600}$ at $4 \mathrm{~h}$ (Fig. 1D and F).

We next investigated expression levels of heterogeneous protein in $H d 1, H d 2, H d 3$, and $H d 4$, no significant difference was observed between the phage-resistant strains and the parental strain (Fig. 1I). This data indicated that the process of mutagenesis and selection did not influence the heterogeneous gene expression machinery in E. coli.

To test whether the engineered strain had a practical benefit in preventing the infection of phage, $H d 1$ and $H d$ NAMPT were chosen for incubation in the incubator room where the phage 1 was collected. Flask containing cells was sealed with a wet gauze-cotton plug that is normally avoided since it increases the probability of contamination compared with dry plugs. All flasks seeded with $\mathrm{Hd} 1$ successfully reached $\mathrm{OD}_{600}$ values larger than 1.5 after $10 \mathrm{~h}$ (Fig. 2). In contrast, $\mathrm{OD}_{600}$ values in eight of ten flasks seeded with Hd NAMPT were lower than 0.3.

\section{The resistant strain generated by mutagenesis and selection contains multiple subclones}

ARTP is an efficient tool to generate diverse microbial mutations, we then investigated whether the resistant strains generated by our method were pure populations. Upon the ARTP treatment, cells were resuspended in $50 \mathrm{ml}$ LB medium and seed into a 96-deep well plate immediately ( $0.5 \mathrm{ml}$ per well). After 6 $\mathrm{h}$ with sharking, $10 \mathrm{ul}$ of 10 -diluted phage 1 was added into each well. Interestingly, cell growths were observed in all wells containing mutagenized cells but not wild-type cells after $16 \mathrm{~h}$ of adding phage 1 (Fig. 3A and B), suggesting that laboratory/factory-specific phage-resistant strains generated by our standard protocol were composed by multiple colonies. The high diversity of population may partly explain why $\mathrm{Hd}^{1}$ can resistant to multiple phages (Fig. 1E), even when that phages have not been encountered.

\section{Our practical method for engineering phage-resistant strains is not associated with desired heterogeneous proteins in $\mathrm{E}$. coli}

To prove the versatility of our method for engineering phage-resistant strains, we added phages into two other mutagenized recombinant strains (Mut3 and GFP). Mut3 was used as a nitrilase whole cell catalyst for synthesis of (R)-(-)-mandelic acid (Zhang et al. 2014). In agreement with the aforementioned result using the Hd NAMPT, Mut3-1, Mut3-2, Mut3-3, Mut3-4 were successfully achieved after incubation of ARTP mutagenized Mut3 with phage1, phage2, phage3, and phage 4, respectively. SDS-PAGE exhibited that the expression levels of nitrilase have not been affected in these phage-resistant strains (Fig. 4A), proving that our practical method for engineering phage-resistant strains was not associated with heterogeneous gene of interest in $\mathrm{E}$. coli. Interestingly, in contrast to $\mathrm{HdNampt}$ derived phage -resistant strains, none of these Mut3 derived strains showed comparable broad anti-phage activities (Fig. 4B-E). It 
could be meaningful to reveal the mechanism underlying the difference, but is beyond the scope of this study.

Finally, we investigated the effect of mutagenesis and selection on the function of heterogeneous protein. GFP1, GFP2, GFP3, and GFP4 were generated for preventing phage1, phage2, phage3, and phage4 attack, respectively. These four stains as well as their parental strain GFP were at the same range of relative fluorescence units (Fig. 4F).

\section{Discussion}

E. coli is used as the most important model organism in the production of valuable substances including vectors, proteins, and bulk chemicals. In the vast majority of these processes, single colonies were used. However, the low complex of bacterial community makes processes vulnerable to phage infection (Flores et al. 2011). For dairy industry, starter cultures containing multiple production strains that are resistant to different phages allow the formation of products even if certain phages exist (Marco et al. 2012). Our data indicated that the same approach can be applied in the operation of $E$. coli through mutagenesis, as the mutagenized populations can resist multiple phages while maintaining the ability to overexpress heterogeneous proteins.

Given its easy operation, rapid mutation, and environmental safety (Zhang et al. 2014), ARTP was used for construction of the mutant library. However, the main disadvantage of ARTP is that a special equipment is required. It remains to investigate whether other mutagenesis methods such UV radiation or chemical mutagens are also available for generating phage-resistant cells.

Bacteria have been attacked by phages throughout evolution. To survive, they have developed various anti-phage systems (Kronheim et al. 2018). Investigation of the evolutionary arms race between bacteria and phages has led to the identification of novel tools such as CRISPR/CAS9 system (Cong and Zhang 2015). However, our knowledge of the battle between bacteria and phages remains poor. The phageresistant strains of $E$. coli generated by our practical method may also provide valuable materials for theoretical research, considering that $E$. coli is the most studied bacteria.

\section{Declarations}

\section{Acknowledgements}

This research was supported by the Young Talents Program of National High-level Personnel of Special Support Program, the Fundamental Research Funds for the Provincial Universities of Zhejiang (RFC2020002), and the Major Research program of Zhejiang Provincial National Natural Science Foundation of China (LD21C050001).

Funding: 
This research was supported by the Young Talents Program of National High-level Personnel of Special Support Program, the Fundamental Research Funds for the Provincial Universities of Zhejiang (RFC2020002), and the Major Research program of Zhejiang Provincial National Natural Science Foundation of China (LD21C050001).

Conflicts of interest/Competing interests:

The authors declare that they have no conflict of interest.

Availability of data and material:

The codon-optimized Haemophilus ducreyi nicotinamide phosphoribosyltransferase with a C-terminal six histidine tag was deposited at GenBank (Accession No: MW759281).

Code availability: Not applicable.

Authors' contributions:

YPX, YGZ, QS, JMX, and ZQL initiated and supervised the project. QS, XTZ, and QG carried out all the experiments and data analyses. QS and YPX are responsible for the preparation and revision of the manuscript. All authors read and approved the final manuscript.

Ethics approval: Not applicable.

Consent to participate: Not applicable.

\section{References}

1. Baeshen MN et al (2015) Production of Biopharmaceuticals in E. coli: Current Scenario and Future Perspectives. J Microbiol Biotechnol 25:953-962. doi:10.4014/jmb.1412.12079

2. Baltz RH (2018) Bacteriophage-resistant industrial fermentation strains: from the cradle to CRISPR/Cas9. J Ind Microbiol Biotechnol 45:1003-1006. doi:10.1007/s10295-018-2079-4

3. Cheng $L$ et al (2020) An antiphage Escherichia coli mutant for higher production of L-threonine obtained by atmospheric and room temperature plasma mutagenesis. Biotechnol Prog 36:e3058. doi:10.1002/btpr.3058

4. Cong L, Zhang F (2015) Genome engineering using CRISPR-Cas9 system. Methods Mol Biol 1239:197-217. doi:10.1007/978-1-4939-1862-1_10

5. Flores CO, Meyer JR, Valverde S, Farr L, Weitz JS (2011) Statistical structure of host-phage interactions. Proc Natl Acad Sci U S A 108:E288-E297. doi:10.1073/pnas.1101595108

6. Kronheim $S$ et al (2018) A chemical defence against phage infection. Nature 564:283-286. doi:10.1038/s41586-018-0767-x 
7. Labrie SJ, Samson JE, Moineau S (2010) Bacteriophage resistance mechanisms. Nat Rev Microbiol 8:317-327. doi:10.1038/nrmicro2315

8. Li P, Lin H, Mi Z, Xing S, Tong Y, Wang J (2019) Screening of Polyvalent Phage-Resistant Escherichia coli Strains Based on Phage Receptor. Analysis Front Microbiol 10:850. doi:10.3389/fmicb.2019.00850

9. Los M (2012) Minimization and prevention of phage infections in bioprocesses. Methods Mol Biol 834:305-315. doi:10.1007/978-1-61779-483-4_19

10. Marco MB, Moineau S, Quiberoni A (2012) Bacteriophages dairy fermentations Bacteriophage 2:149-158. doi:10.4161/bact.21868

11. Rostol JT, Marraffini L (2019) (Ph)ighting Phages: How Bacteria Resist Their Parasites. Cell Host Microbe 25:184-194. doi:10.1016/j.chom.2019.01.009

12. Shen Q et al (2020) Engineering a Pichia pastoris nitrilase whole cell catalyst through the increased nitrilase gene copy number and co-expressing of ER oxidoreductin 1. Appl Microbiol Biotechnol 104:2489-2500. doi:10.1007/s00253-020-10422-4

13. Wang C, Pfleger BF, Kim SW (2017) Reassessing Escherichia coli as a cell factory for biofuel production. Curr Opin Biotechnol 45:92-103. doi:10.1016/j.copbio.2017.02.010

14. Wang T, Zhang $X$, Bheda P, Revollo JR, Imai S, Wolberger $C$ (2006) Structure of Nampt/PBEF/visfatin, a mammalian NAD + biosynthetic enzyme. Nat Struct Mol Biol 13:661-662. doi:10.1038/nsmb1114

15. Zhang X, Zhang XF, Li HP, Wang LY, Zhang C, Xing XH, Bao CY (2014) Atmospheric and room temperature plasma (ARTP) as a new powerful mutagenesis tool. Appl Microbiol Biotechnol 98:5387-5396. doi:10.1007/s00253-014-5755-y

\section{Figures}


A

phage 1

phage2
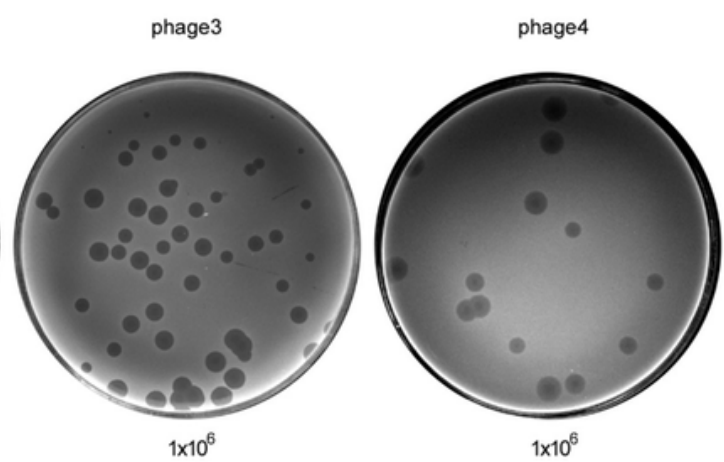

Dilution:
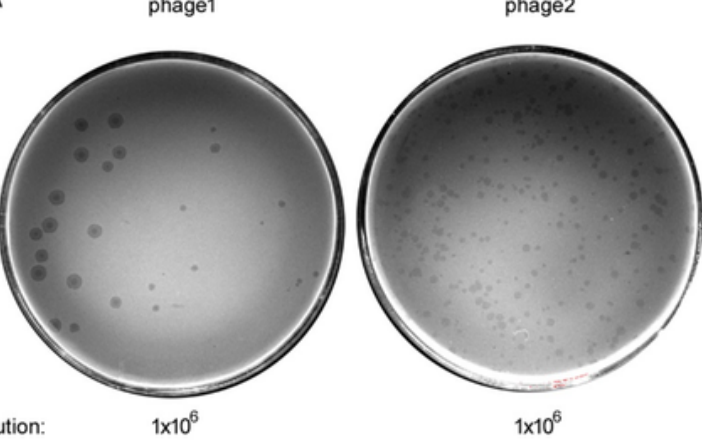

$1 \times 10^{6}$

$1 \times 10^{6}$

B

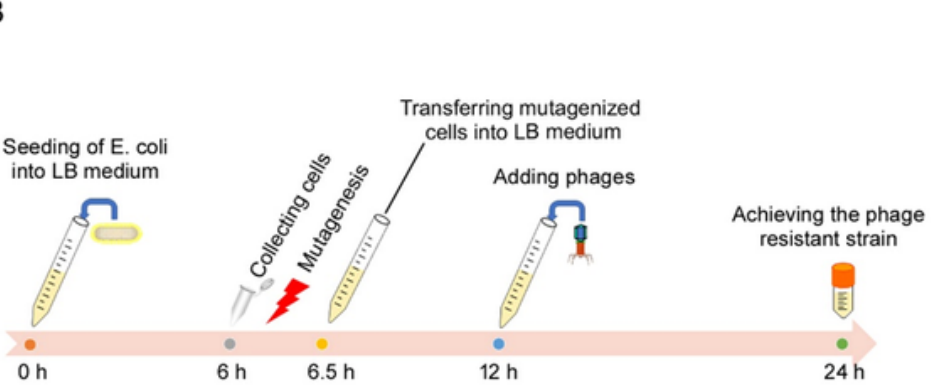

C

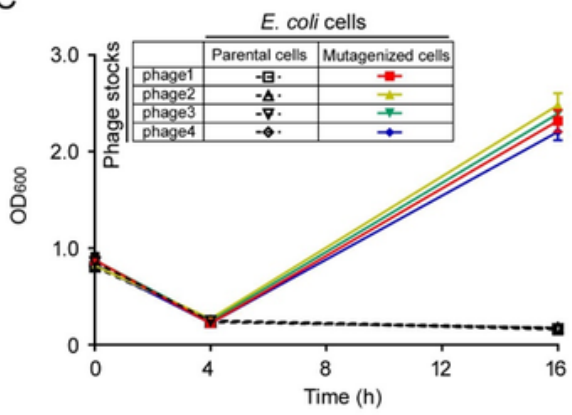

D

E

F
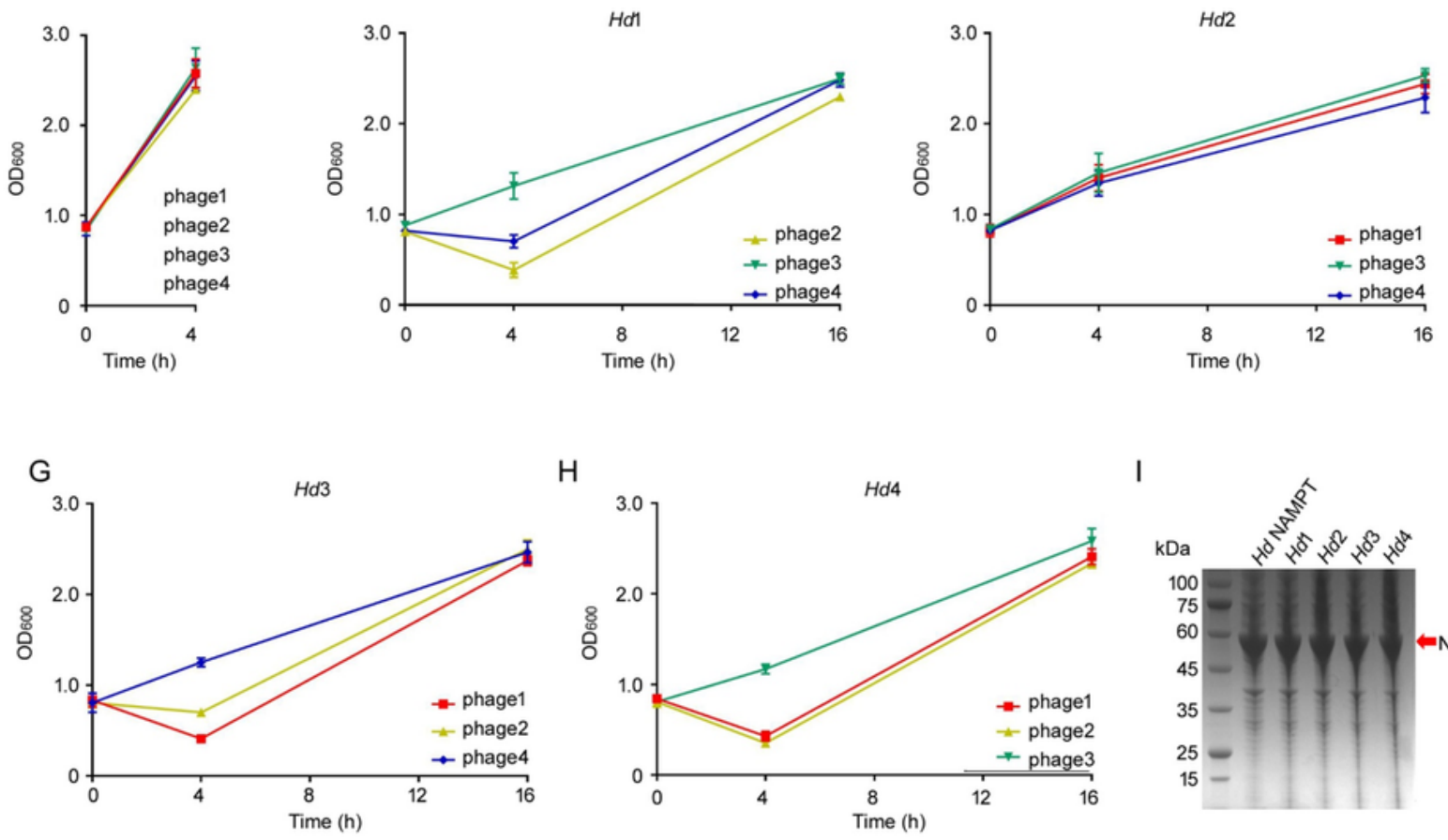

$\mathrm{H}$
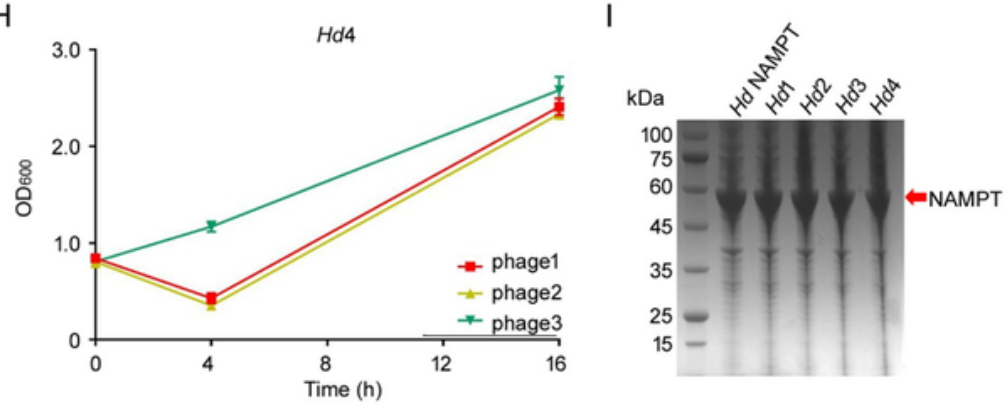

\section{Figure 1}

Generation of phage-resistant Hd NAMPT. (A) The plaque assay of phage1, phage2, phage3, and phage4. Phage stocks were diluted one million-fold. (B) The workflow for generation laboratory/factory-specific phage-resistant strains. (C) Subpopulations of mutagenized cells can resist the infection of phages. (D) Resistant strains acquired immunity to the phages that they have encountered. The resistance spectrum 
of $\mathrm{Hd} 1(\mathrm{E}), \mathrm{Hd} 2(\mathrm{~F}), \mathrm{Hd} 3(\mathrm{G})$, and $\mathrm{Hd} 4(\mathrm{H})$ to indicated phages. (I) The expression levels of NAMPT in $\mathrm{Hd}$ NAMPT, Hd1, Hd2, Hd3, and Hd4.

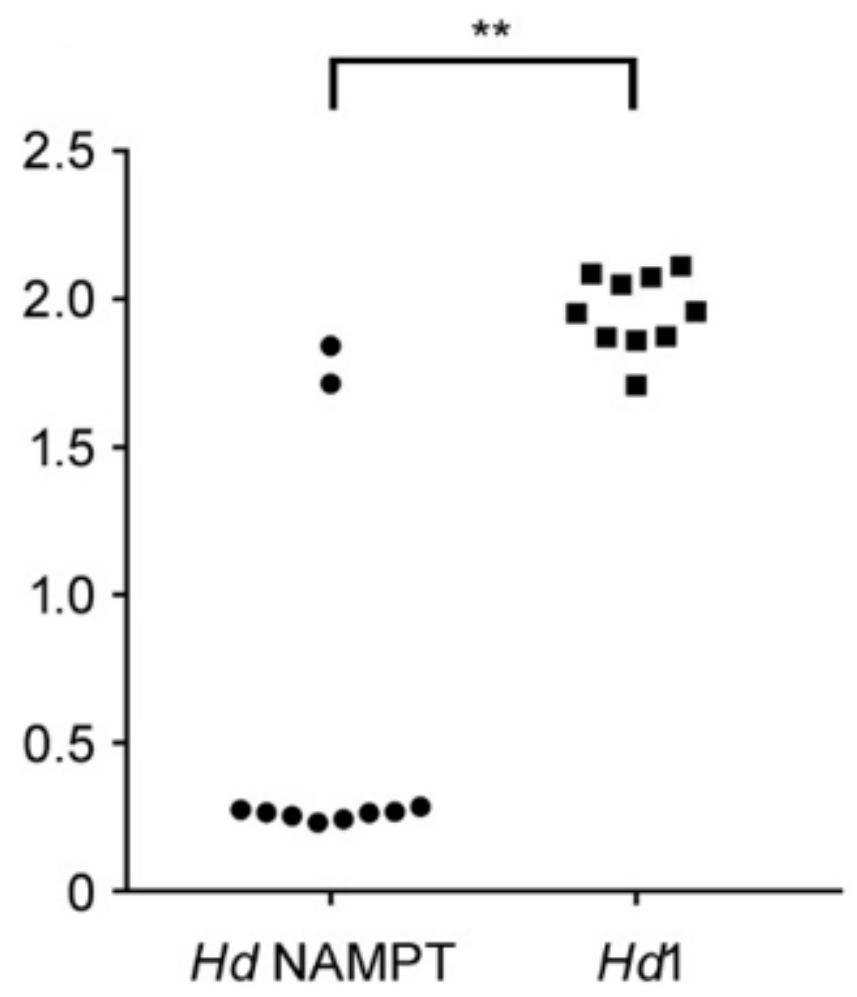

Figure 2

Engineered strain decreased the chances of phage contamination. Hd NAMPT and Hd1 were seed into flasks sealed with wet gauze-cotton plugs. All flasks were shaken in the incubator room where the phage 1 was collected. OD600 values were measured after $10 \mathrm{~h}$ of seeding. 
Parental cells growth in the absence of phage1

Parental cells growth in the presence of phage1

\section{OD600}
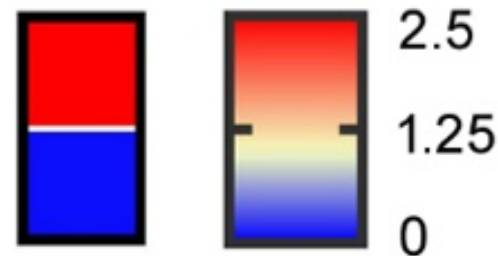

B

ARTP mutagenized cells growth in the presence of phage1

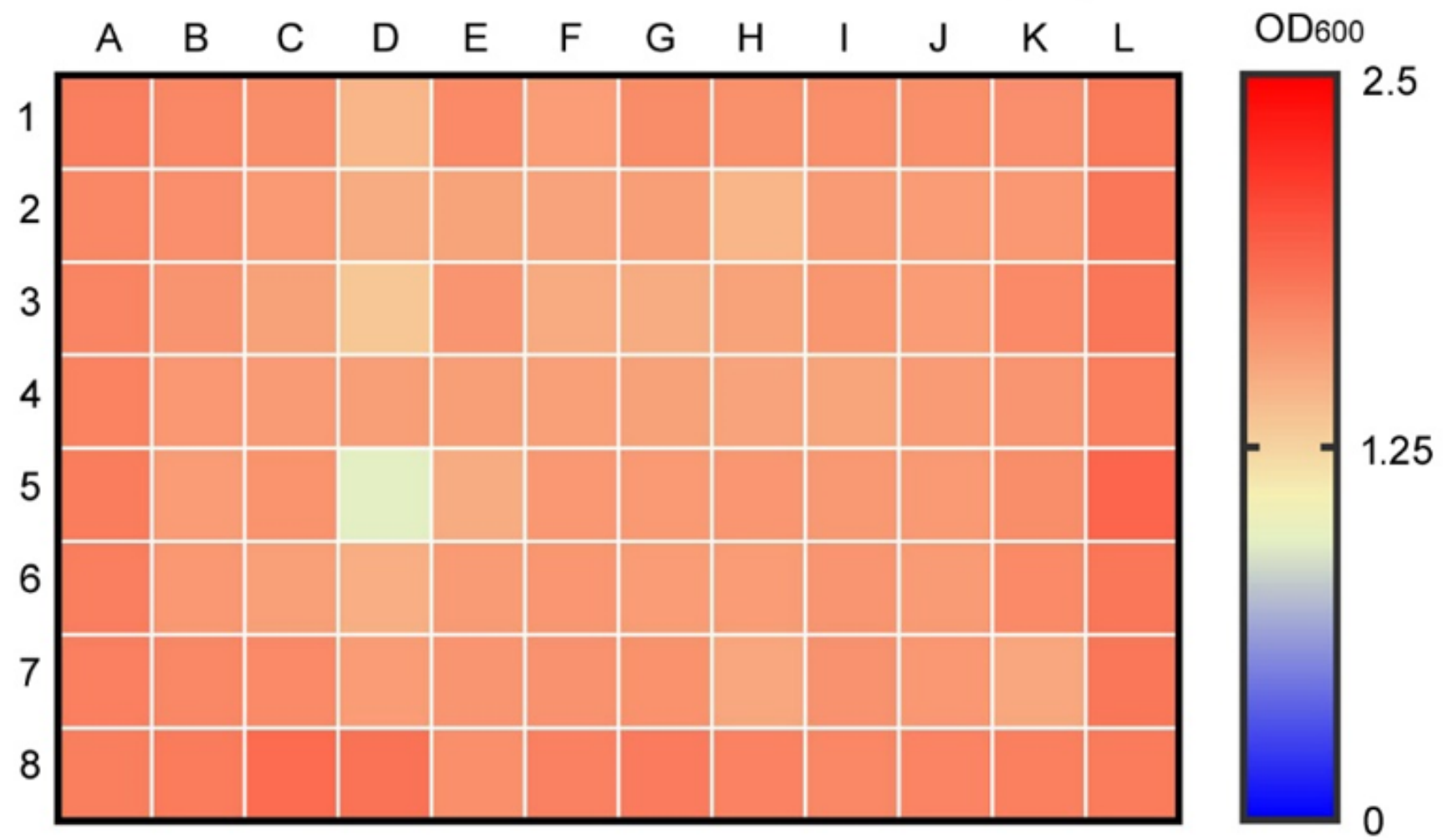

Figure 3

The resistant strain generated by mutagenesis and selection contains multiple subclones. (A) Growth of Hd NAMPT in the presence or absence of phage1. OD600 values were measured after $16 \mathrm{~h}$ of seeding. (B) Growth of ARTP mutagenized cells in the presence of phage1. Cells were seeded into 96-deep well plate immediately after mutagenesis. Phage 1 was added into each well $4 \mathrm{~h}$ of seeding. OD600 values were measured after $16 \mathrm{~h}$ of adding phage 1. 
A

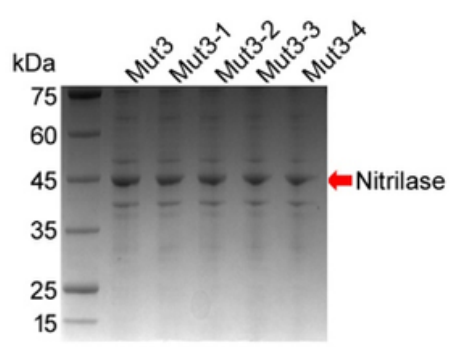

B

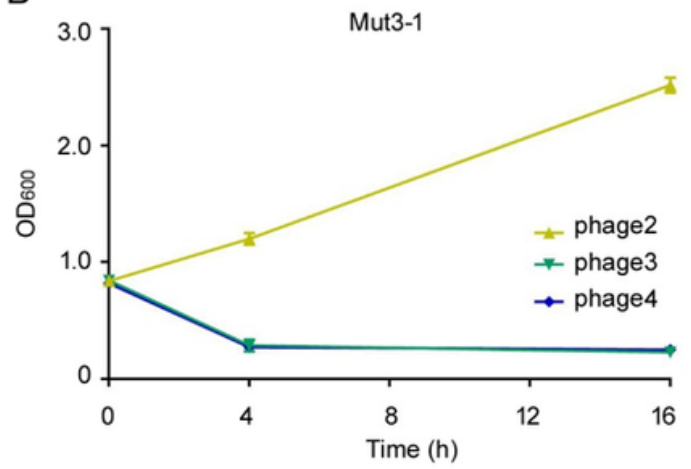

E
C

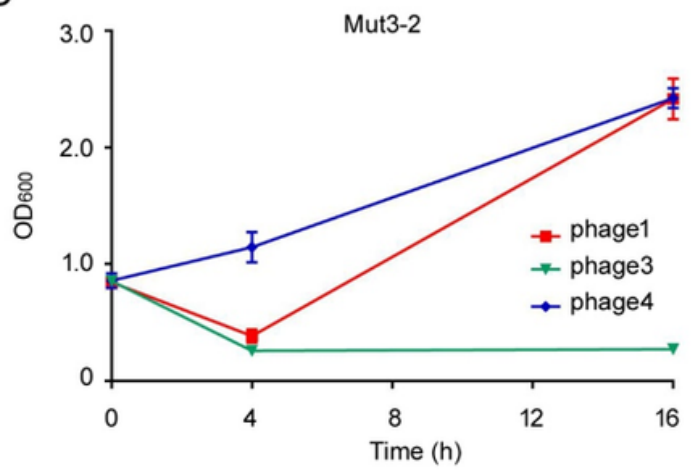

$\mathrm{F}$
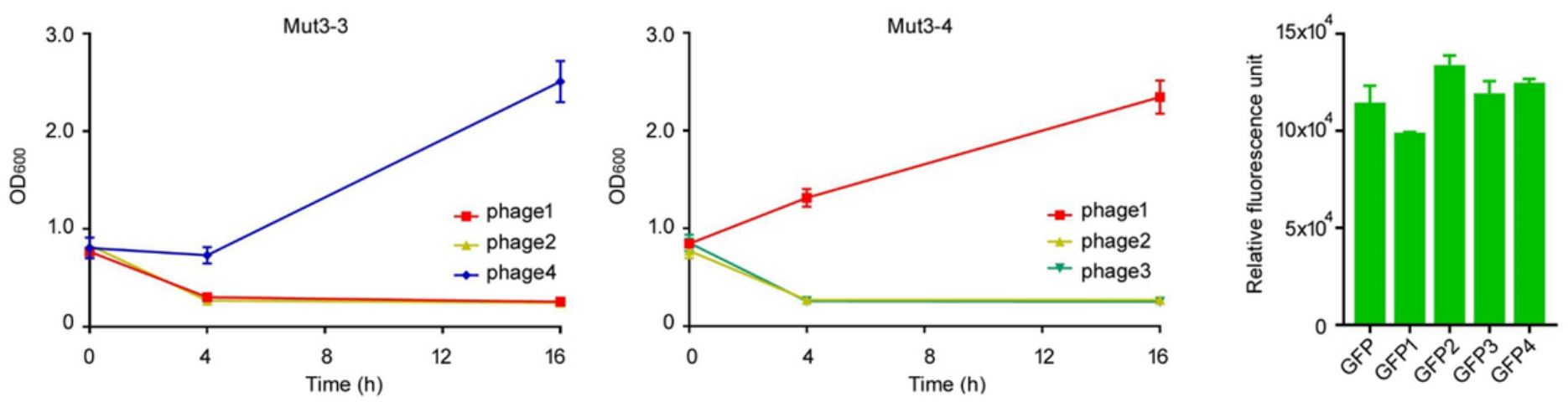

Figure 4

Generation of phage-resistant Mut3 and GFP. (A) The expression levels of nitrilase in Mut3, Mut3-1, Mut32, Mut3-3, and Mut3-4. (B) The resistance spectrum of Mut3-1 (B), Mut3-2 (C), Mut3-3 (D), and Mut3-4 (E) to indicated phages. (F) Relative fluorescence units of GFP, GFP1, GFP2, GFP3, and GFP4. 